\title{
Optical S-ALOHA/CDMA Systems for Multirate Applications: Architecture, Performance Evaluation, and System Stability
}

\author{
Robert Raad, Member, IEEE, Elie Inaty, Member, IEEE, Paul Fortier, Senior Member, IEEE, and \\ Hossam M. H. Shalaby, Senior Member, IEEE
}

\begin{abstract}
In this paper, a new multirate optical network based on a hybrid slotted-ALOHA (S-ALOHA)/overlapped code division multiple access (O-CDMA) system is proposed as an effective way of integrating multiclass traffic. The key issue in this proposal is to exploit the potential of the optical O-CDMA using fiber Bragg gratings when jointly used with the S-ALOHA protocol in a link layer. The newly proposed system is modeled using a general Markov chain from which both the system throughput and the average packet delay are derived. The system stability passing through a general finite user model is also envisaged. The system is then compared to the classical S-ALOHA/variable processing gain (VPG) CDMA system. Numerical results show that the proposed system outperforms the latter one, especially at high transmission rates. In addition, stability measures demonstrate that the overlapped system tends to be more stable than the VPG system.
\end{abstract}

Index Terms-Fiber Bragg grating (FBG), first exit time (FET), multirate, optical link layer, overlapped code division multiple access (O-CDMA), slotted ALOHA, variable bit rate, variable processing gain (VPG) code division multiple access (CDMA).

\section{INTRODUCTION}

$\mathbf{O}$ WING to the rapid progress in fiber-optic technology, optical code division multiple access (CDMA) utilizing fiber Bragg gratings (FBGs) is gaining more interest in the creation of all-optical communication systems for integrating heterogeneous traffic sharing a single broadband optical channel with a multiplicity of quality of service (QoS) and traffic requirements [4], [10], [11]. In addition, the bursty characteristics of high-speed data traffic in optical local area networks (LANs) should yield benefits for CDMA technologies that are capable of allocating a high number of simultaneous resources in a decentralized way while increasing the system throughput and decreasing the average packet delay. Slotted-ALOHA (S-ALOHA) techniques could fulfill these requirements when integrated with the newly proposed optical overlapped fast frequency hopping CDMA (O-FFH-CDMA) system [5].

Although there is a lot of research in the field of optical CDMA, which has been done until now, most of it has

Manuscript received September 15, 2005; revised January 24, 2006.

R. Raad and E. Inaty are with the Department of Computer Engineering, University of Balamand, El-Koura, North Lebanon (e-mail: robert.j.raad@ balamand.edu.lb; elie.inaty@balamand.edu.lb).

P. Fortier is with the Department of Electrical and Computer Engineering, Laval University, Laval, Quebec City, QC G1K 7P4, Canada (e-mail: fortier@gel.ulaval.ca).

H. M. H. Shalaby is with the Department of Electrical Engineering, University of Alexandria, Alexandria 21544, Egypt (e-mail: shalaby@ieee.org).

Digital Object Identifier 10.1109/JLT.2006.872684 focused on the physical layer [1], [3], [5], [11]. This fact also applies to the works that have been conducted in the area of multirate optical CDMA. A multirate optical FFHCDMA system using variable processing gain (VPG) has been proposed in [4]. The intention was to guarantee the one-to-one correspondence between the PG and the source transmission rate. The drawback of this system is the drastic decrease in the transmitted signal power, especially for higher rate users for which the PG becomes very small. The solution to this problem is the use of power control. On the other hand, in [1] and [2], multilength frequency-hopping codes are considered. Using these codes, the rate and QoS are now dynamically matched to users' needs. The cutoff rate of the system is still limited by the physical constraints of the codes. Lately, we have considered the overlapped CDMA (O-CDMA) system [5], where we have shown that it is possible to increase a class bit rate beyond the nominal rate without decreasing the PG of the desired user or allowing any time delay between the data symbols.

Many researches have been conducted on S-ALOHA and random-access CDMA [6], [9], [12]. Nevertheless, jointly used with O-CDMA, random-access packet switching becomes a challenging issue [7], [8], [10], [16] to cope with the increasing packet-type demand with large population, shorter messagedelay delivery, and minimum packet-rejection probability. In [7] and [8], protocols for a high-speed fiber-optic LAN using passive star topology were proposed. New optical CDMA access protocols with and without pretransmission coordination were studied in [10]. On the other hand, Xue et al. [16] were concerned with two optical CDMA random-access schemes that support tunable transmitter/fixed receiver and fixed transmitter/tunable receiver, respectively. In this paper, we propose a new hybrid system that combines a variable-transmission-rate optical O-FFH-CDMA system [5] with the S-ALOHA protocol (S-ALOHA/O-FFH-CDMA) as a novel and simple scheme of achieving multiuser and multirate capability in decentralized optical CDMA packet networks. In particular, a variable transmission rate is achieved by increasing the bit rate beyond the nominal limits while keeping the PG and the time-slot duration unchanged. This yields overlapping among bits in a single time slot. Thus, varying the amount of overlapping bits can vary the transmission rate.

Following the introduction, this paper is structured as follows. Section II introduces the system model, which employs the discrete-time Markov chain describing the system states. 


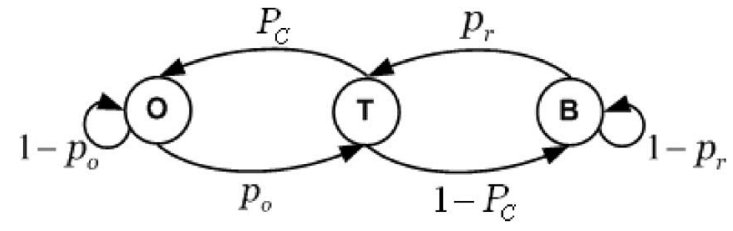

Fig. 1. Traffic model.

The signal-to-interference ratio (SIR) is derived in Section III. Section IV presents the performance evaluation of the system. Stability measures are discussed in Section V. Numerical results are covered in Section VI. Finally, concluding remarks end the paper in Section VII.

\section{SySTEM MODEL}

We consider an optical FFH-CDMA communication network that supports $K$ terminals, which share the same optical medium in a star architecture [11]. The encoding and decoding are achieved passively using a sequence of FBGs. The gratings will spectrally and temporally slice an incoming broadband pulse into several components equally spaced at chip intervals $T_{c}$ [11]. The chip duration and the number of gratings will establish the nominal bit rate of the system, i.e., the round-trip time of light, from a given transmitted bit, to be totally reflected from the encoder. This nominal bit duration in a structure of $G$ gratings is given by $T_{n}$, where $G$ is the PG. The corresponding nominal rate is $R_{n}=1 / T_{n}=1 / G T_{c}$.

Using the aforementioned passive network, we propose a hybrid S-ALOHA/FFH-CDMA optical packet network in which each user is assigned a unique code, which is characterized by zero autocorrelation property using a frequency-shifted version (FSV) proposed in [11], and a cross correlation between any two different codewords of at most one. Subsequently, up to $K$ users can simultaneously transmit $K$ packets in a given time slot.

The traffic model used in this paper is the general Markov chain, in which the traffic is distributed according to the user's activity, as shown in Fig. 1. It is called a general arrival model.

Each of the $K$ terminals can be in one of the three operational modes at a time, i.e., origination mode, transmission mode, or backlog mode. The user in the origination mode generates and transmits a new packet at the beginning of the next time slot with a probability $P_{o}$. The user enters the backlog mode when an attempt to transmit a new packet fails. This event occurs with probability $1-P_{c}$, where $P_{c}$ is the correct packet probability of the corresponding system. The retransmissions of a backlogged packet occur in any given time slot with probability $P_{r}$. In the backlogged mode, the blocked terminal cannot generate new packets until the backlogged packet is received correctly. According to the variations of $P_{o}$ and $P_{r}$, we obtain the following cases.

1) For $P_{r}=P_{o}=p$, the arrival distribution can be approximated by either a binomial distribution if the number of users $K$ in the system is finite or by a Poisson distribution if $K$ is very high. For both distributions, the average offered load is approximated by $R=K P_{o}$.
2) For $P_{r}>P_{o}$, it is very difficult to obtain a closed-form approximation for the arrival distribution. Thus, we use the general Markov model to track the flow of packets in the system based on the user activity without any approximation. In this case, the offered load is in its maximum since it includes the fraction of the retransmitted packets. Note that 1) is considered as a particular case of 2).

3) For $P_{r}<P_{o}$, the interpretation is similar to case 2); however, the offered load is diminishing, and there is a high probability that the system queue will overflow, and some backlogged packets will be lost. It is not a practical case especially in ultrahigh-speed optical systems.

For practical implementations, we assume that the priority of retransmitting the backlogged packets is always higher than that of generating new packets, and the traffic distribution is based on the current user's activities, so it is general. Henceforth, case 2 ) is adopted in our analysis.

Fig. 2 represents the model of the proposed optical SALOHA/FFH-CDMA system. The streams of composite arrivals of this system consist of $\xi_{o}$ newly generated packets plus $\xi_{r}$ retransmitted packets.

The statistical behavior of the terminal can be described using a general discrete Markov chain [6]. The system state represents the number of backlogged terminals $n$.

Before continuing the analysis, let us impose some restrictions that help in simplifying the mathematical evaluations and in improving the clarity of the problem under consideration. We assume 1) a synchronous system and discrete rate variation, 2) a single-class system; and 3) unit transmission power for all the users.

In this paper, the transmission rate of the terminals $R_{s}$ is allowed to be greater than $R_{n}$ according to two different methods, as explained below.

\section{A. S-ALOHA/VPG-FFH-CDMA System}

Assume a fixed packet time duration of $T_{p}=L T_{n}=L G T_{c}$, where $L$ is the nominal packet length. In this system, the variable transmission rate is accomplished by varying the PG $G_{V}$ in such a way that increasing the transmission rate by a factor of $\alpha \geq 1$ allows the reduction of spreading factor by the same amount $G_{V}=(G / \alpha)$ [4]. The bit rate in this case is given by

$$
R_{s}=\alpha R_{n}(\mathrm{bits} / \mathrm{s})
$$

In a packet network, $X_{b}^{(V)}=\lfloor\alpha L\rfloor$ bits are allocated in a time slot instead of $L$, as shown in Fig. 3, where $\lfloor x\rfloor$ is the highest integer less than $x$. Then, the new transmission rate becomes

$$
R_{s}=\frac{X_{b}^{(V)}}{L} R_{n}(\mathrm{bits} / \mathrm{s}) .
$$

In Fig. 3(a), we present a case study where $G_{V}=5$ and $L=2$, which means the nominal rate is 2 bits/packet. On the other hand, in Fig. 3(b), we have decreased the PG to $G_{V}=3$ (which means $\alpha=5 / 3$ ) in order to increase the transmission rate to 3 bits/packet. 


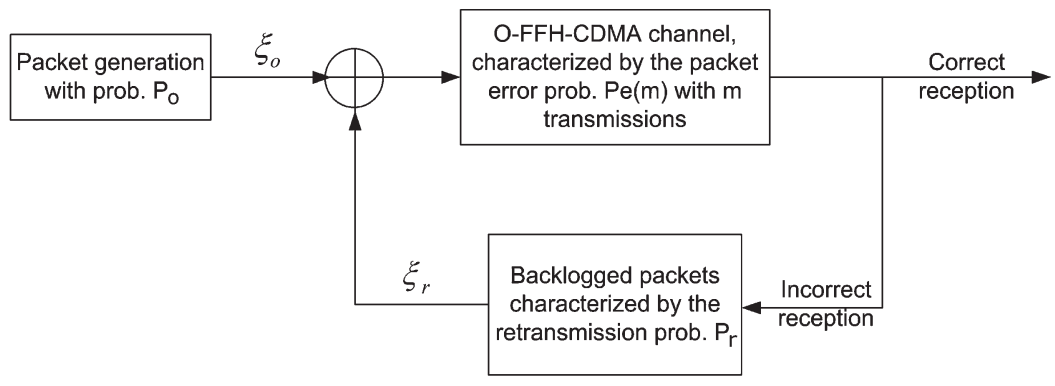

Fig. 2. Optical S-ALOHA/FFH-CDMA system model.

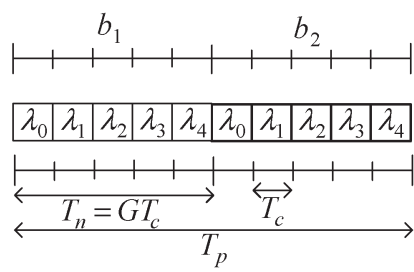

(a)

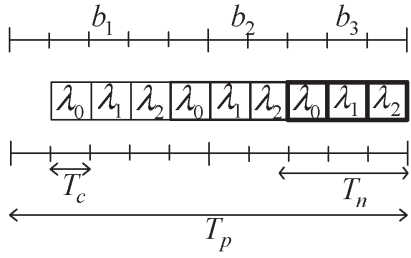

(b)
Fig. 3. Optical S-ALOHA/VPG-FFH-CDMA packet model of a single user in a given packet time slot. (a) $G_{V}=5$. (b) $G_{V}=3$.

\section{B. S-ALOHA/O-FFH-CDMA System}

In [5], we have shown that due to the linearity of the encoder-decoder set, multibits will be coded and transmitted when the data rate increases beyond $R_{n}$, as shown in Fig. 4. At the receiver end, the decoder practically observes multicodes, which are delayed according to the transmission rate of the source.

Accordingly, in the S-ALOHA/O-FFH-CDMA, to increase the number of bits per packet of fixed length $L$, we increase the source transmission rate above the nominal rate without decreasing the PG as in the previous system. When a terminal transmits using a rate $R_{s}>R_{n}$, it introduces a bit overlap coefficient $\varepsilon_{s}$, which represents the number of overlapping chips between two consecutive bits [5]. Accordingly, the new bit rate is related to $R_{n}$ throughout the following equation:

$$
R_{s}=\frac{G}{G-\varepsilon_{s}} R_{n} .
$$

Let $\varepsilon_{s}$ be the overlapping coefficient and $X_{b}^{(O)}$ be the total number of overlapped bits in a packet time slot. For an overlapped packet to be complete for transmission, the following inequality must be satisfied:

$$
\begin{aligned}
& \varepsilon_{s}+\underbrace{\left(G-\varepsilon_{s}\right)+\cdots+\left(G-\varepsilon_{s}\right)}_{X_{b}^{(O)} \text { times }} \leq L G \\
& X_{b}^{(O)} \leq \frac{L G-\varepsilon_{s}}{G-\varepsilon_{s}} .
\end{aligned}
$$

Thus

$$
X_{b}^{(O)}=\left\lfloor\frac{L G-\varepsilon_{s}}{G-\varepsilon_{s}}\right\rfloor .
$$

Consequently, the rate in a packet network will be

$$
R_{s}=\frac{X_{b}^{(O)}}{L} R_{n}(\mathrm{bits} / \mathrm{s})
$$

Fig. 4 illustrates an example of the overlapping process in a packet time slot. In this example, the packet length is $L=2$, and the PG is $G=5$. If the transmission rate is the nominal rate, the packet format is as shown in Fig. 3(a), which means $\varepsilon_{s}=0$ and the transmission rate is 2 bits/packet. When the overlapped coefficient is increased to $\varepsilon_{s}=3$, as shown in Fig. 4(a), the transmission rate is increased to 3 bits/packet. On the other hand, Fig. 4(b) shows the case where $\varepsilon_{s}=4$. Accordingly, the transmission rate is 6 bits/packet.

The relation between the reduction factor $\alpha$ of the SALOHA/VPG-FFH-CDMA system and the overlapping coefficient $\varepsilon_{s}$ of the S-ALOHA/O-FFH-CDMA system can be easily obtained by equating (2) and (7) to obtain

$$
\alpha=\frac{G-\frac{\varepsilon_{s}}{L}}{G-\varepsilon_{s}} .
$$

\section{Signal-To-INTERFERENCE RATIO (SIR)}

In this section, we derive the SIR for both systems. In general, we can write the SIR as follows:

$$
\mathrm{SIR}=\frac{G^{2}}{\sum_{k=1}^{K-1} \sigma_{I_{k}}^{2}+\sigma_{n}^{2}}
$$

where $\sigma_{I_{k}}^{2}$ is the interference power from the $k$ th terminal, and $\sigma_{n}^{2}$ is the additive white Gaussian noise (AWGN) power. The SIR for both systems differ in $\sigma_{I_{k}}^{2}$ and the PG.

\section{A. S-ALOHA/VPG-FFH-CDMA System}

Assume class- $s$ users with transmission rate $R_{s}$ and equivalent PG $G_{V}$. Also, assuming equally probable data, we can write the interference power [4] as follows:

$$
\sigma_{I_{k}}^{2}=\sum_{q=0}^{G_{V}-1}\left[H_{k}^{2}(0, q)+H_{k}^{2}\left(q, G_{V}\right)\right]
$$




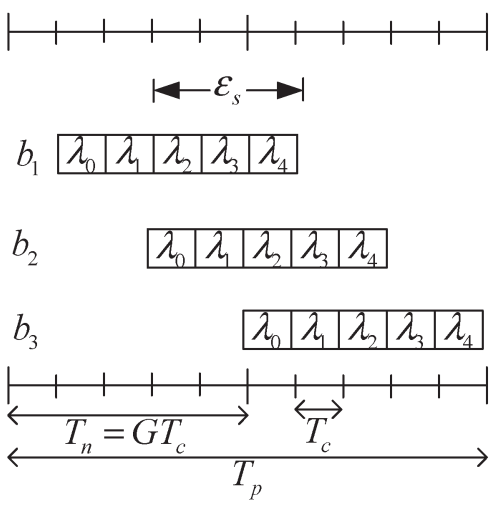

(a)

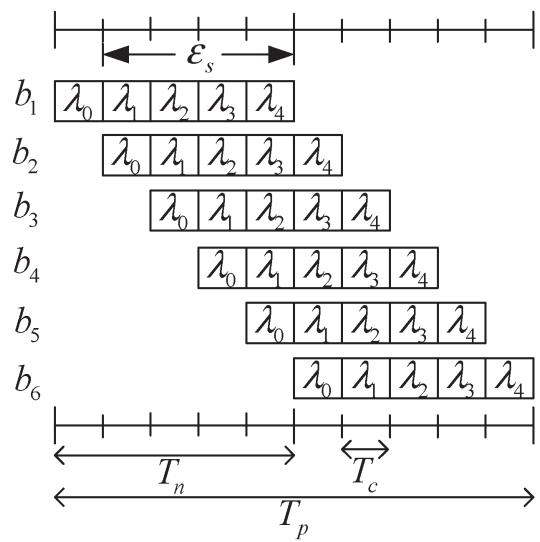

(b)

Fig. 4. Optical S-ALOHA/O-FFH-CDMA packet model of a single user in a given time slot. (a) $\varepsilon_{s}=3$. (b) $\varepsilon_{s}=4$.

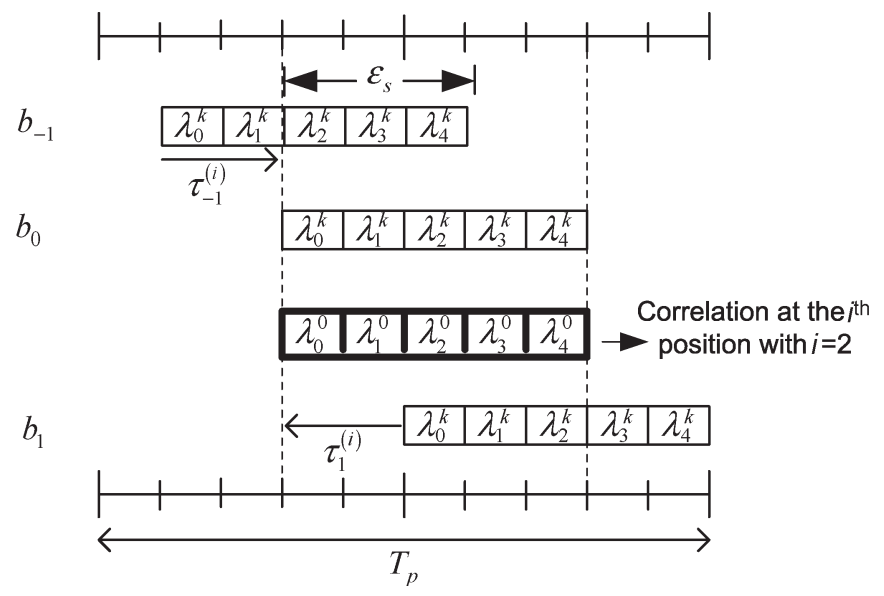

Fig. 5. Interference from user $k$ on the desired user due to overlapping.

where

$$
H_{k}(a, b)=\sum_{j=a}^{b} h\left(a_{j-a}^{k}, a_{j}^{0}\right)
$$

is the discrete-time partial-period Hamming cross-correlation function of the $k$ th interferer [4]. In addition, $h(\cdot)^{1}$ is the Hamming function [4]. The sequences $a_{j-q}^{k}$ and $a_{j}^{0}$ are numbers representing frequencies of the $k$ th interferer and the desired user, respectively. Notice that the interference power is equal for every bit in the packet time slot. Thus, we can write

$$
\operatorname{SIR}^{(i)}=\frac{\frac{G^{2}}{\alpha^{2}}}{\sum_{k=1}^{K-1} \sigma_{I_{k}}^{2}+\sigma_{n}^{2}}, \quad \forall 1 \leq i \leq X_{b}^{(V)}
$$

where $\alpha$ is the reduction factor given in (1).

$$
{ }^{1} h(a, b)= \begin{cases}0, & a \neq b \\ 1, & a=b\end{cases}
$$

\section{B. S-ALOHA/O-OFFH-CDMA System}

In the S-ALOHA/O-OFFH-CDMA system, the problem is much more complex due to the fact that not every bit in the packet time slot will have the same SIR as shown in Fig. 5. Therefore, the SIR at the $i$ th time-slot position is given by

$$
\operatorname{SIR}^{(i)}=\frac{G^{2}}{\sum_{k=1}^{K-1} \sigma_{I_{k}}^{2}(i)+\sigma_{n}^{2}}, \quad \forall 1 \leq i \leq X_{b}^{(O)}
$$

where $X_{b}^{(O)}$ is given by (6) and $\sigma_{I_{k}}^{2}(i)$ can be computed as follows. Letting $X_{r}=\left\lceil\varepsilon_{s} /\left(G-\varepsilon_{s}\right)\right\rceil$, where $\lceil x\rceil$ is the smallest integer greater than $x$, we can notice that the problem can be divided into two parts.

1) $X_{b}^{(O)}-2 X_{r} \leq 0$ :

$$
\sigma_{I_{k}}^{2}(i)= \begin{cases}\sum_{\nu=-i}^{-1} H_{\nu}^{2}\left(0, q_{\nu}^{(i)}\right) & \\ & +\sum_{\nu=0}^{X_{r}} H_{\nu}^{2}\left(q_{\nu}^{(i)}, G\right), \quad 0 \leq i \leq X_{b}^{(O)}-X_{r}-1 \\ & \sum_{\nu=-i}^{-1} H_{\nu}^{2}\left(0, q_{\nu}^{(i)}\right) \\ & +\sum_{\nu=0}^{X_{b}^{(O)}-i-1} H_{\nu}^{2}\left(q_{\nu}^{(i)}, G\right), \quad X_{b}^{(O)}-X_{r} \leq i \leq X_{r}-1 \\ & \\ & \sum_{\nu=-X_{r}}^{-1} H_{\nu}^{2}\left(0, q_{\nu}^{(i)}\right) \\ & \\ +X_{b}^{(O)}-i-1 & \\ & \end{cases}
$$

where $q_{\nu}^{(i)}=\tau_{\nu}^{(i)} / T_{c}$, and $\tau_{\nu}^{(i)}$ is the time delay with respect to the $i$ th bit in the packet. $\left(j-q_{\nu}^{(i)}\right)$ is evaluated modulo $G$. 
2) $X_{b}^{(O)}-2 X_{r}>0$ :

$$
\sigma_{I_{k}}^{2}(i)= \begin{cases}\sum_{\nu=-i}^{-1} H_{\nu}^{2}\left(0, q_{\nu}^{(i)}\right) & \\ +\sum_{\nu=0}^{X_{r}} H_{\nu}^{2}\left(q_{\nu}^{(i)}, G\right), & 0 \leq i \leq X_{r}-1 \\ \sum_{\nu=-X_{r}}^{-1} H_{\nu}^{2}\left(0, q_{\nu}^{(i)}\right) & \\ +\sum_{\nu=0}^{X_{r}} H_{\nu}^{2}\left(q_{\nu}^{(i)}, G\right), & X_{r} \leq i \leq X_{b}^{(O)}-X_{r}-1 \\ \sum_{\nu=-X_{r}}^{-1} H_{\nu}^{2}\left(0, q_{\nu}^{(i)}\right) & \\ +\sum_{\nu=0}^{X_{b}-i-1} H_{\nu}^{2}\left(q_{\nu}^{(i)}, G\right), & X_{b}^{(O)}-X_{r} \leq i \leq X_{b}^{(O)}-1 .\end{cases}
$$

\section{Performance Evaluation}

\section{A. Packet Error Probability}

If a Gaussian hypothesis is used to model the interference, the probability of bit error in a time slot when there are $K$ simultaneous active terminals using a simple ON-OFF Keying (OOK) modulation is related to the system's SIR using the following equation:

$$
P_{b}(i)=Q\left(\frac{1}{2} \sqrt{\operatorname{SIR}^{(i)}}\right)
$$

where $\operatorname{SIR}^{(i)}$ is given in (12) or in (13).

1) S-ALOHA/VPG-FFH-CDMA System: Due to the fact that the bit error probability is equal for every bit in the packet, the probability of successfully receiving a packet is

$$
P_{c}(K)=\left[1-P_{b}(i)\right]^{X_{b}^{(V)}}
$$

where $P_{b}(i)$ is given in (16).

2) S-ALOHA/O-FFH-CDMA System: On the other hand, for the S-ALOHA/O-FFH-CDMA system, the bit error probability is not equal for every bit in the packet. It depends on the bit position $i$, as revealed in (14) and (15). Thus, we can write

$$
P_{c}(K)=\prod_{i=0}^{X_{b}^{(O)}-1}\left[1-P_{b}(i)\right]
$$

\section{B. System Throughput and Average Packet Delay}

Let $P=\left[P_{n m}\right]$ be the transition matrix, where $P_{i j}$ is the onestep transition probability from state $i$ to state $j$. Consider that $P_{n m}$ is the probability that $m$ backlogged users will be present in the next state given that $n$ are present in the current state. It is given by

$$
P_{n m}=\operatorname{Pr}\{x(t+1)=m \mid x(t)=n\} .
$$

For random-access CDMA, transition can take place in a number of ways since there can be more than one successful transmission per time slot. The transition from state $n$ to state $m$ is determined by the difference between the number of unsuccessful new transmission UNTX and successful retransmission SRTX, i.e., when SRTX exceeds UNTX by $(n-m)$, for $n \geq$ $m$, or UNTX exceeds SRTX by $(m-n)$, for $m \geq n$. Let $\mathrm{NTX}=\xi_{o}$ be the number of new transmissions and RTX $=\xi_{r}$ be the number of retransmissions. Let $b(\psi, \delta, p)$ denote the binomial distribution. It characterizes the total number of all possible $\psi$ successes in any order given $\delta$ attempts, and $p$ is the probability of success. Then, the joint probability distribution of SRTX and UNTX given $n$ users in the backlogged mode can be written as

$$
\begin{aligned}
\operatorname{Pr} & \{\mathrm{SRTX}=k, \mathrm{UNTX}=l \mid x(t)=n\} \\
& =\sum_{\substack{\xi_{o}=l \\
0 \leq l \leq K-n \\
0 \leq k \leq n}}^{K-n} \sum_{\xi_{r}=k}^{n}\left\{b\left[l, \xi_{o}, P_{E}\left(\xi_{o}+\xi_{r}\right)\right] \cdot b\left[\xi_{o}, K-n, P_{o}\right]\right.
\end{aligned}
$$$$
\left.\cdot b\left[k, \xi_{r}, P_{c}\left(\xi_{o}+\xi_{r}\right)\right] \cdot b\left[\xi_{r}, n, P_{r}\right]\right\}
$$

where $b(\psi, \delta, p)$ is given by

$$
b(\psi, \delta, p)=\left(\begin{array}{l}
\psi \\
\delta
\end{array}\right) p^{\delta}(1-p)^{\psi-\delta}
$$

and $P_{c}(\cdot)$ is the correct packet probability and given by (18) and (29). In addition, $P_{E}(\cdot)=1-P_{c}(\cdot)$, which represents the packet error probability.

Hence, the steady-state transition probability is calculated as follows:

$P_{n m}= \begin{cases}\sum_{j=0}^{\min (n, K-m)} \operatorname{Pr}\left\{\begin{array}{l}\operatorname{SRTX}=j, \\ \mathrm{UNTX}=m-n+j \mid x(t)=n\end{array}\right\}, & m \geq n \\ \sum_{j=0}^{\min (m, K-n)} \operatorname{Pr}\left\{\begin{array}{l}\mathrm{SRTX}=n-m+j, \\ \mathrm{UNTX}=j \mid x(t)=n\end{array}\right\}, & m \leq n .\end{cases}$

The long-term state occupancy probability $\mu(n)$ is given by the solution of

$$
\mu^{\mathrm{T}}=\mu^{\mathrm{T}} P, \quad \mu^{\mathrm{T}}=\left[\begin{array}{lll}
\mu(0) & \cdots & \mu(n)
\end{array}\right], \quad \sum_{n=0}^{K} \mu(n)=1 .
$$

The method to solve (23) is to find the eigenvalues of $P^{\mathrm{T}}$ and their corresponding eigenvectors. By sorting the $(K+1)$ eigenvalues in ascending order, the desired eigenvector is the one whose index is the index of the highest eigenvalue. Then, the long-term state probability vector will correspond to the desired eigenvector divided by the sum of their entries.

Thus, the probability of success can be seen as a binomial distribution by which $S$ packets are received successfully given $M$ attempted transmissions in a given time slot. This leads to the following:

$$
\operatorname{Pr}(S=s \mid M=m)=b\left[s, m, P_{c}(m)\right] .
$$


The steady-state composite arrival distribution given that there are $n$ backlogged users in the system and $m$ backlogged users in the next time slot is given by

$$
\begin{aligned}
& f_{M}(m \mid x=n) \\
& \quad=\sum_{j=\max (m-n, 0)}^{\min (m, K-n)} \operatorname{Pr}(\mathrm{NTX}=j, \mathrm{RTX}=m-j \mid x=n) \\
& \quad=\sum_{j=\max (m-n, 0)}^{\min (m, K-n)} b\left(j, K-n, P_{o}\right) \times b\left(m-j, n, P_{r}\right) .
\end{aligned}
$$

The throughput in packet per time slot $\beta$ is defined as the expected number of successful transmissions per time slot

$$
\begin{aligned}
\beta & =E\{S\}=E\{E\{S \mid M\}\} \\
& =E\left[\sum_{s=0}^{K} s\left(\begin{array}{c}
M \\
s
\end{array}\right) P_{c}^{s}\left(1-P_{c}\right)^{M-s}\right] \\
& =\sum_{m=1}^{K} m P_{c}(m)\left[\sum_{n=0}^{K} f_{M}(m \mid n) \mu(n)\right] .
\end{aligned}
$$

The offered traffic can be estimated by

$$
R=(K-\bar{n}) P_{o}+\bar{n} P_{r}
$$

where $\bar{n}$ is the expected backlog or the average system state and is given by

$$
\bar{n}=\sum_{n=0}^{K} n \mu(n) .
$$

The steady-state delay according to Little's theorem can be written as

$$
D=\frac{\bar{n}}{\beta}
$$

\section{Stability Measures}

Stability analysis has considerable interest for assessing the performance of ALOHA systems [6], [9], [14], [15]. According to Kleinrock and Lam [9], a stable S-ALOHA system exists when the channel load line intersects nontangentially the equilibrium throughput-delay contour by a single point called system operating point $\left(\beta_{o}, \bar{n}_{o}\right)$; otherwise, the system is unstable. Then, the authors introduce the stability measure as the average first exit time (FET) into the unsafe region where the average number of backlogged users $\bar{n}$ starts increasing exceeding a given threshold $\bar{n}_{O}$ and the system's throughput begins vanishing, assuming the system is initiated with no backlogged users. In [15], system stability is evaluated in terms of the expected drift in state $n$, which represents the expected deviation of the system state from its current state to any other state. Thus, the system remains in its current state, and when changing, it moves to lower states avoiding saturation.
Therefore, the expected drift is the measure of the dynamics of the S-ALOHA system, and it is defined as

$$
d(n)=\sum_{m=0}^{K}(m-n) P_{n m} .
$$

A stable system has a global stable equilibrium point at state $n=\bar{n}_{o}$ with zero drift and a monotonically decreasing slope. In this case, the system has an acceptable throughput rate and a tolerable time delay. The equilibrium points with zero drift and monotonically increasing slope make the system unstable. The system that has more than one equilibrium point is a bistable system [15].

It is shown by simulation (next section) that it is computationally very difficult to estimate the relative degree of stability between the overlapped and the VPG systems by using the expected drift analysis. The FET stability measure offers a clear idea about the useful operating time of both systems in the safe region before exiting to the unsafe region with much lower computational complexity.

Let the random variable $T_{i}$ be the number of transitions the channel backlog $\bar{n}$ goes through before it surpasses $\bar{n}_{0}$ for the first time starting from state $i$. Therefore, FET is denoted by the expected value $\bar{T}_{0}$ of $T_{i=0}$, which is obtained by solving the set of linear equations

$$
\bar{T}_{i}=1+\sum_{j=0}^{\bar{n}_{o}} P_{i j} \bar{T}_{j}
$$

where $P_{i j}$ is the transition probability from state $i$ to state $j$. The solution of the linear system is derived as

$$
\overline{\boldsymbol{T}}=(\boldsymbol{P}-\boldsymbol{I})^{-1} \times(-\mathbf{1})
$$

where $\boldsymbol{P}$ is the transition matrix, $\boldsymbol{I}$ is the identity matrix, and $\mathbf{1}$ is a column vector of ones. FET is then evaluated as

$$
\bar{T}_{0}=\sum_{j=0}^{\bar{n}_{o}} \bar{T}_{j} P_{0 j} .
$$

$\bar{n}_{o}$ corresponds to the maximum throughput $\beta_{o}$ such that the system is stable when $\bar{n}<\bar{n}_{o}$, and unstable otherwise. The stability of both systems for a finite population model has been studied numerically herein.

\section{NUMERICAL RESULTS}

Throughout this part, we present simulation results using the system model previously derived in Sections III-V. Our target is to compare the network throughput, the average packet delay, and the stability of the S-ALOHA/O-FFH-CDMA and the S-ALOHA/VPG-FFH-CDMA systems. Before presenting the simulation results, it is worthwhile to summarize the procedures adopted throughout this section. This is summarized as follows.

1) Specify the total number of terminals $K$.

2) Assign each terminal a code using the extended hyperbolic congruential (EHC) family of codes [13]. 


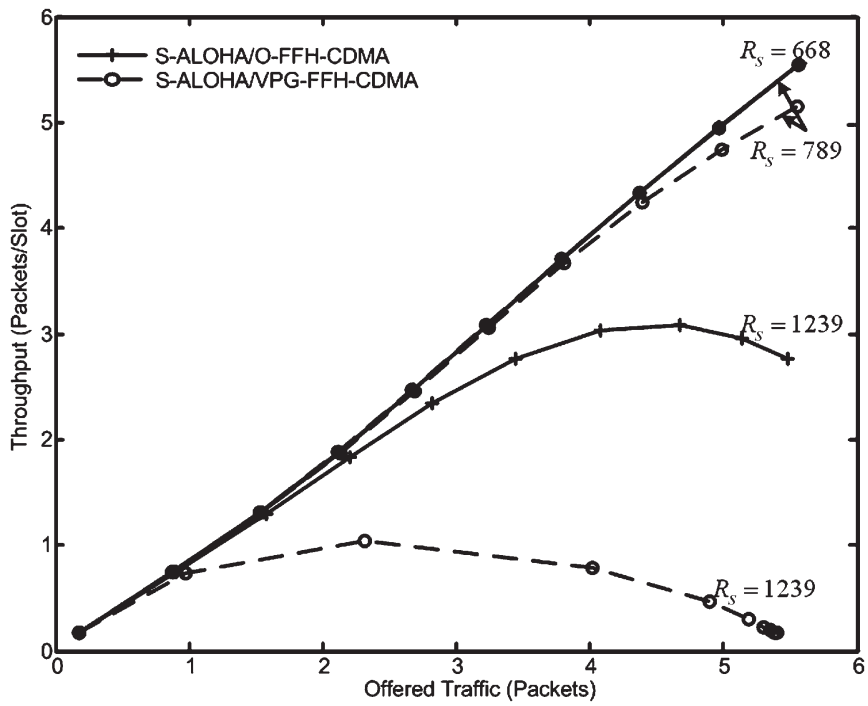

Fig. 6. Throughput versus offered traffic for $P_{r}=0.9$.

3) Choose the transmission rate of each terminal $R_{s}$ by specifying the PG $G_{V}$ for the S-ALOHA/VPG-FFHCDMA throughout (2) and by specifying the overlapping coefficient $\varepsilon_{s}$ for the S-ALOHA/O-FFH-CDMA throughout (7).

4) Targeting a specific terminal, we use a correlation receiver to compute the multiple access interference (MAI) power from each undesired terminal using the derived equations (10), (14), and (15).

5) The SIR is therefore computed using (9), (12), and (13).

6) Then, we can compute the probability of error and the packet correct probability using (16)-(18).

7) The packet correct probabilities are used in the $n$-state Markov model in (20), (24), and (26) in order to obtain the state transition probabilities, the throughput, and the average packet delay, respectively.

8) The computed transition probability matrix is used in (30) and (32) in order to obtain the stability analysis.

9) This process is done for both the S-ALOHA/VPG-FFHCDMA and the S-ALOHA/O-FFH-CDMA system, and a comparison is performed.

In the simulations, we have taken the number of active terminals $K=6$, the packet length $L=300$ bits/time slot, and $G=29$. In addition, the transmission rate $R_{s}$ is normalized in the sense that it represents the number of bits per time slot $T_{p}$.

In the upcoming simulations, we consider three different transmission bit rates, $R_{s}=668,789$, and 1239 bits/time slot, for both overlapped and VPG systems. For the S-ALOHA/OFFH-CDMA, we will assume that the bits in a packet have overlapping coefficients of $\varepsilon_{s}=16,18$, and 22 chips. This, in turn, yield increases in the bit rates to $R_{s}=668,789$, and 1239 bits/time slot, respectively. On the other hand, for the S-ALOHA/VPG-FFH-CDMA, the equivalent increases in the bit rates to $R_{s}=668,789$, and 1239 bits/time slot is achieved by reducing the PG to $G_{V}=13,11$, and 7 , respectively.

First, assume that the probability of retransmission is taken to be $P_{r}=0.9$. In Fig. 6 , we plot the throughput versus the offered traffic for the three different values of $R_{s}$. Notice that at

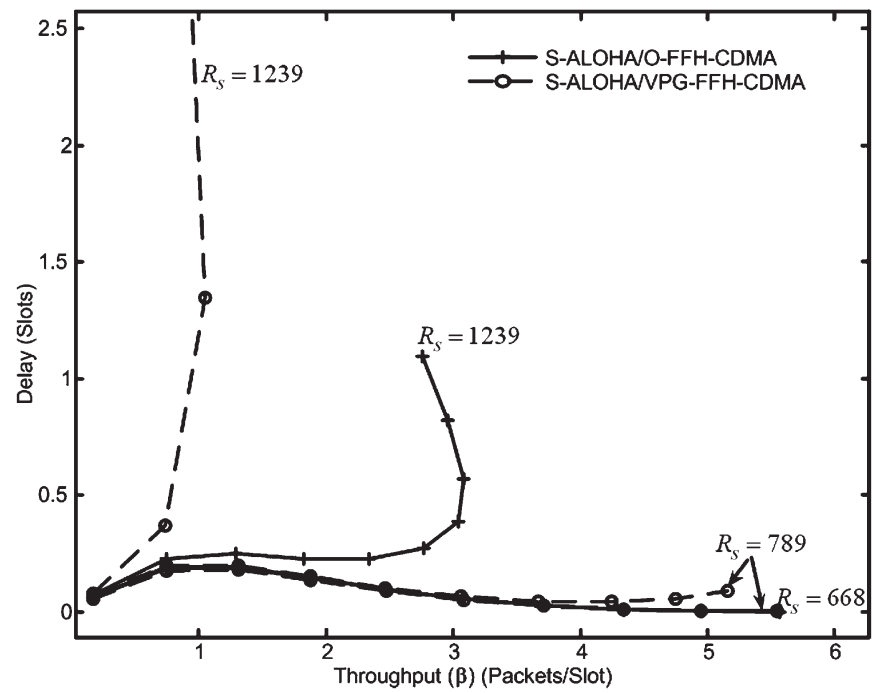

Fig. 7. Average delay versus throughput for $P_{r}=0.9$.

a high bit rate, it is evident that when the offered load increases, the throughputs of both systems degrade rapidly because they are governed by the SIR at the physical layer, as in (9) and (12). In fact, as the SIR declines, the packets are more vulnerable to distortion, which reduces the likelihood of their successful transmission and reception. Thus, the packet correct probabilities in (17) and (18) collapse, and hence, the throughput, while at low and moderate rates, is relatively higher. However, the overlapped system can tolerate more traffic than VPG.

On the other hand, Fig. 7 shows the average delay versus the throughput for the three different values of $R_{s}$. Since at low and moderate bit rates both systems can handle high loads, their throughputs are considerably higher. Therefore, rarely do backlogged packets occur and the systems' queuing delays are negligible. Nevertheless, as the bit rate increases to higher values, the overall interference significantly degrades the system throughput. In this case, a large portion of the offered load is corrupted and backlogged packets are delayed for retransmission. However, since the systems operate at a high rate, the retransmitted packets might face the same destiny, and then, they will be further delayed.

It is clear that for both performance measures, the $\mathrm{S}$ ALOHA/O-FFH-CDMA system always outperforms the SALOHA/VPG-FFH-CDMA system and for different $R_{s}$. Notice that as the transmission rate increases, the S-ALOHA/OFFH-CDMA becomes much better than the S-ALOHA/VPGFFH-CDMA in the sense that its throughput becomes much higher and its delay becomes much smaller. This result is expected because when the transmission rate becomes very high, the PG becomes very small for the S-ALOHA/VPG-FFHCDMA system. This, in turn, drastically decreases the SIR in (12), and therefore, the packet correct probability in (17). On the other hand, for the S-ALOHA/O-FFH-CDMA system, the PG is fixed, which means that the signal power remains the same. In addition, the overlapping coefficient is increased, which in turns slightly increases the MAI, as revealed in (14) and (15). It seems that the slight increase in the MAI induced by the overlapping procedure in the S-ALOHA/O-FFH-CDMA system is much better than the huge decrease in the signal 


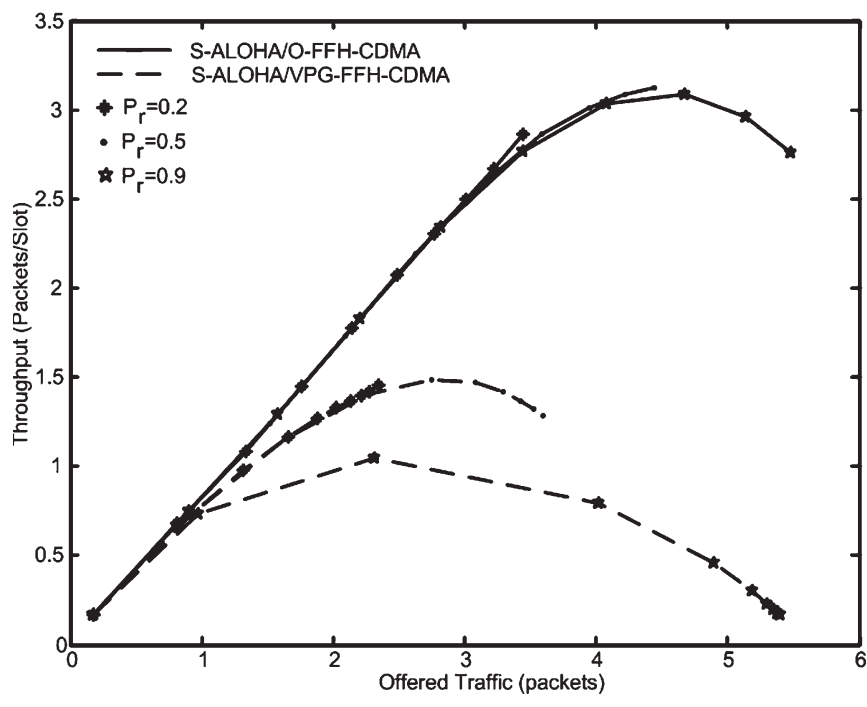

Fig. 8. Throughput versus offered traffic for $R_{s}=1239$ bits/slot.

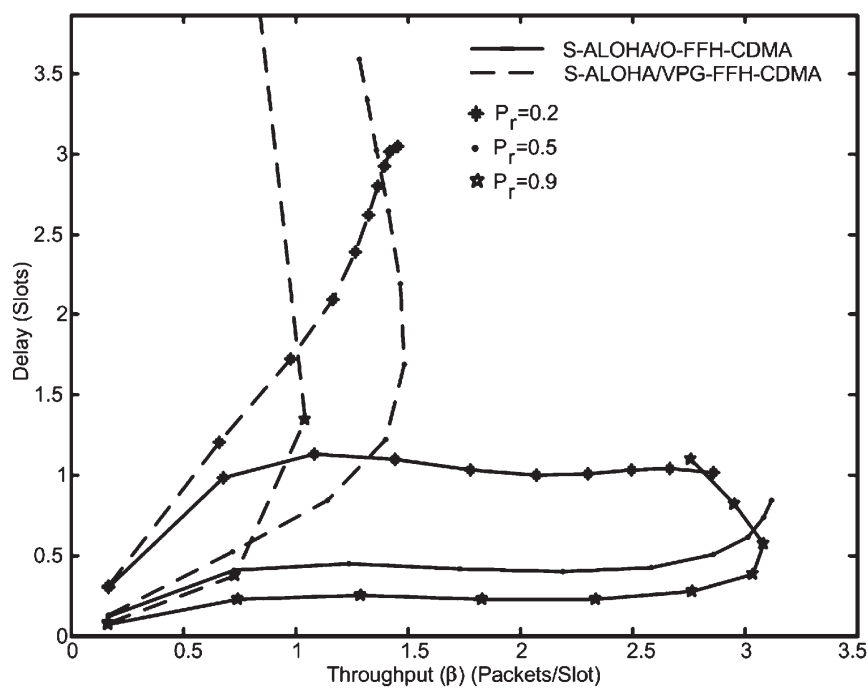

Fig. 9. Average delay versus throughput for $R_{s}=1239$ bits/slot.

power in the S-ALOHA/VPG-FFH-CDMA system in terms of keeping the SIR high enough to achieve an acceptable packet correct probability.

The effect of the retransmission probability $P_{r}$ on the system's throughputs and the average packet delay is studied in Figs. 8 and 9 , respectively, by varying $P_{r}$ and fixing $R_{s}$ to 1239 bits/slot. In Fig. 8, we plot the throughput versus the offered traffic, and Fig. 9 shows the average delay versus the throughput. It is clear that as $P_{r}$ decreases, the throughput decreases and the delay increases, which is what we should expect. Note that in Fig. 8, increasing $P_{r}$ from 0.2 to 0.9 yields faster degradation in throughputs of both systems due to the fact that higher priority is now given to the retransmissions, which overload the optical channel. However, in Fig. 9, the average packet delay is radically reduced because the queuing time of backlogged packets is reduced as well. We can clearly notice that the S-ALOHA/O-FFH-CDMA system still outperforms the S-ALOHA/VPG-FFH-CDMA system.

Fig. 10 depicts the state occupancy probability of both the overlapped and the VPG systems. In the latter, it is observed

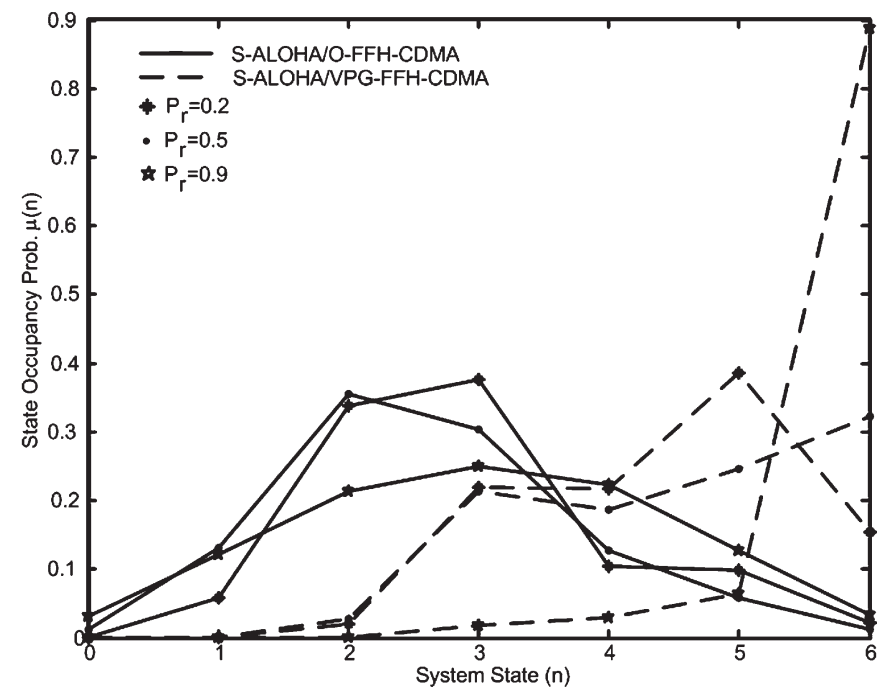

Fig. 10. State occupancy probability versus system states for $R_{s}=$ 1239 bits/slot.

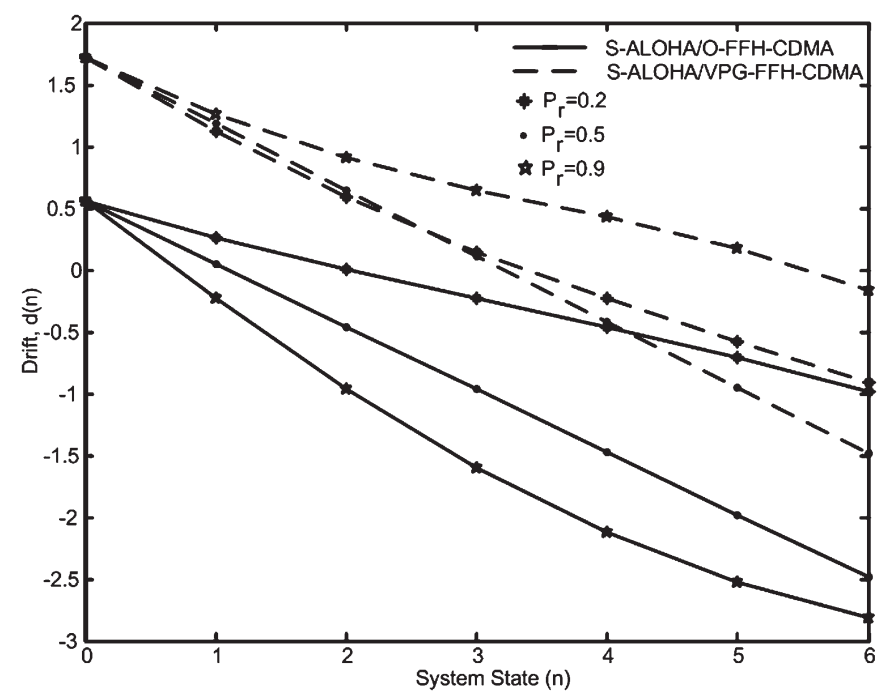

Fig. 11. Expected drift versus system states for $R_{s}=1239$ bits/slot.

that higher order system states are occupying in a monotonically increasing fashion of probability as the retransmission probability increases $\left(P_{r}=0.9\right)$, which lead to more backlogged users in the system, whereas in the former, higher order system states are less probable to occur, and the system converges to the initial state, despite the variation of the retransmissions. This makes the overlapped system tolerate higher rates with acceptable delay.

Fig. 11 shows the expected drift stability assessment of both systems. It is shown that both systems have single global equilibrium points for small number of states. To achieve the second zero crossing point (which is the unstable point), the simulation should be conducted for a much higher number of states, which is numerically very complex.

For this reason, to compare the stability of the overlapped and the VPG systems, the FET stability measure is used as depicted in Fig. 12. It is very clear that the FET for the overlapped system is always higher than that of the VPG system for a different number of active terminals and transmission rates. This implies 


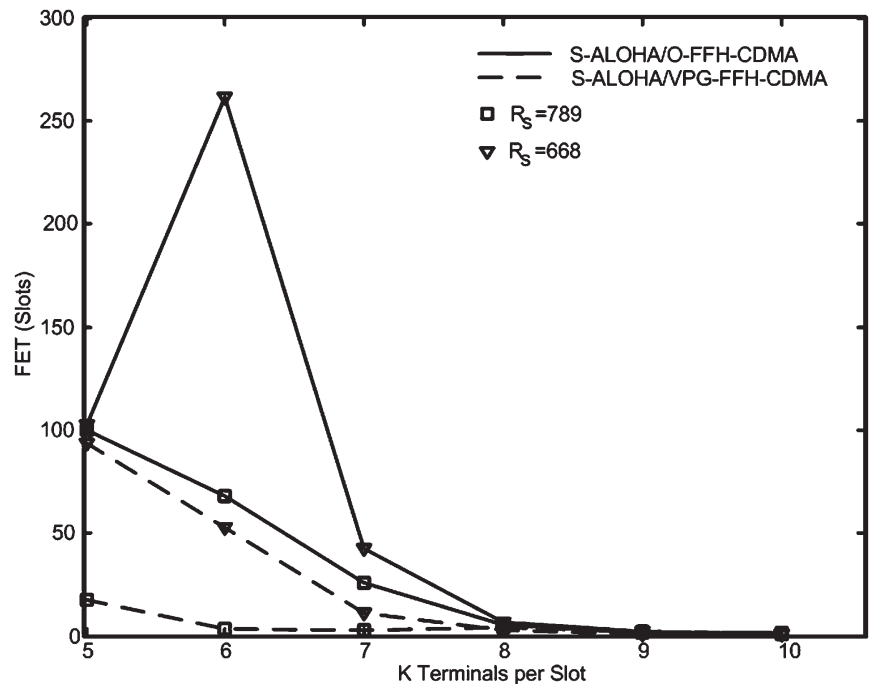

Fig. 12. FET at retransmission probability $P_{r}=0.9$.

that the O-CDMA system is more stable because it operates at a longer time in the safe region before it exits to the unsafe region.

\section{CONCLUSION}

In this paper, a variable-bit-rate system based on a hybrid S-ALOHA/O-FFH-CDMA system was proposed for optical CDMA time-slotted packet networks. Two different systems were introduced and compared-the novel S-ALOHA/O-FFHCDMA system and the classical S-ALOHA/VPG-FFH-CDMA system. The SIR for the new system was obtained. In addition, the time-slotted system model has been derived using the general Markov chain from which the steady-state throughput and average packet delay have been evaluated. Simulation results showed that the newly proposed S-ALOHA/O-FFH-CDMA system outperforms the S-ALOHA/VPG-FFH-CDMA system, especially at higher transmission rates.

In addition, the stability of both systems has been studied in terms of expected drift and FET. Simulation results showed that the newly proposed S-ALOHA/O-FFH-CDMA system is more stable than the S-ALOHA/VPG-FFH-CDMA system. Therefore, it has superior stability and is less dynamic to saturation.

\section{REFERENCES}

[1] J. G. Zhang, "Novel optical fiber code-division multiple access networks supporting real-time multichannel variable-bit-rate (VBR) video distributions," IEEE Trans. Broadcast., vol. 43, no. 3, pp. 339-349, Sep. 1997.

[2] - "Design of a special family of optical CDMA address codes for fully asynchronous data communications," IEEE Trans. Commun., vol. 47, no. 7, pp. 967-973, Jul. 1999.

[3] S. Maric, O. Moreno, and C. J. Corrada, "Multimedia transmission in fiber optical LAN's using optical CDMA,” J. Lightw. Technol., vol. 14, no. 10, pp. 2149-2153, Oct. 1996.

[4] E. Inaty, L. A. Rusch, and P. Fortier, "SIR performance evaluation of a multirate OFFH-CDMA system," IEEE Commun. Lett., vol. 5, no. 5, pp. 224-226, May 2001.

[5] E. Inaty, H. M. H. Shalaby, and P. Fortier, "On the cutoff rate of a multi-rate, multi-class overlapped OFFH-CDMA system," IEEE Trans. Commun., vol. 53, no. 2, pp. 323-334, Feb. 2005.

[6] D. Raychaudhuri, "Performance analysis of random access packetswitched code division multiple access systems," IEEE Trans. Commun., vol. COM-29, no. 6, pp. 895-901, Jun. 1981.
[7] G. N. M. Sudhakar, N. D. Georganas, and M. Kavehrad, "Slotted aloha and reservation aloha protocols for very high-speed optical fiber local area networks using passive star topology," J. Lightw. Technol., vol. 9, no. 10, pp. 1411-1422, Oct. 1991.

[8] P. W. Dowd, "Protocols for very high-speed optical fiber local area network using a passive star topology," J. Lightw. Technol., vol. 9, no. 6, pp. 799-808, Jun. 1991.

[9] L. Kleinrock and S. S. Lam, "Packet switching in a multiaccess broadcast channel: Performance evaluation," IEEE Trans. Commun., vol. COM-23, no. 4, pp. 410-422, Apr. 1975.

[10] H. M. H. Shalaby, "Optical CDMA random access protocols with and without pretransmission coordination," J. Lightw. Technol., vol. 21, no. 11, pp. 2455-2462, Nov. 2003.

[11] E. Inaty, H. M. H. Shalaby, and P. Fortier, "A new transmitter-receiver architecture for noncoherent multirate OFFH-CDMA system with fixed optimal detection threshold," J. Lightw. Technol., vol. 20, no. 11, pp. 1885-1894, Nov. 2002.

[12] F. L. Lo, T. S. Ng, and T. T. Yuk, "Performance analysis of a fullyconnected full-duplex CDMA ALOHA network with channel sensing and collision detection," IEEE J. Sel. Areas Commun., vol. 14, no. 9, pp. 17081716, Dec. 1996.

[13] L. D. Wronski, R. Hossain, and A. Albicki, "Extended hyperbolic congruencial frequency hop code: Generation and bounds for cross- and autoambiguity function," IEEE Trans. Commun., vol. 44, no. 3, pp. 301-305, Apr. 1996.

[14] P. W de Graaf and J. S. Lehnert, "Performance comparison of a slotted ALOHA DS/SSMA network and a multichannel narrow-band slotted ALOHA network," IEEE Trans. Commun., vol. 46, no. 4, pp. 544-552, Apr. 1998.

[15] A. B. Carleial and M. E. Hellman, "Bistable behavior of ALOHA-type systems," IEEE Trans. Commun., vol. COM-23, no. 4, pp. 401-410, Apr. 1975.

[16] F. Xue, Z. Ding, and S. J. B. Yoo, "Performance analysis for optical CDMA networks with random access schemes," in Proc. IEEE Globecom, Nov. 2004, pp. 1883-1887.

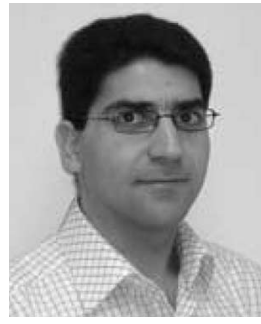

Robert Raad (S'01-M'03) was born in Rahbé, North Lebanon, on August 24, 1978. He received the B.S. and M.S. degrees in computer engineering from the University of Balamand, El-Koura, North Lebanon, in 2001 and 2003, respectively. He is currently working toward the Ph.D. degree at Laval University, Quebec City, QC, Canada.

His research interests include optical code division multiple access (CDMA) and network control and resource-management issues in optical communications networks.

Mr. Raad is the recipient of an award from the professors of the Faculty of Engineering, University of Balamand, El-Koura, Lebanon, in 2003.

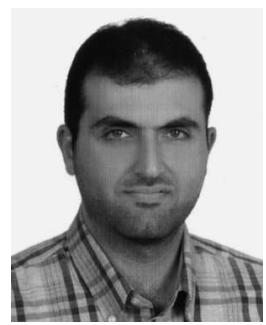

Elie Inaty (S'99-M'02) was born in El-Koura, North Lebanon, on June 23, 1975. He received the B.S. and M.S. degrees in electrical engineering from the University of Balamand, El-Koura, Lebanon, in 1996 and 1998, respectively, and the Ph.D. degree from Laval University, Quebec City, QC, Canada, in 2001.

$\mathrm{He}$ is currently an Assistant Professor at the University of Balamand. His research interests include code division multiple access (CDMA) and wavelength division multiplexing (WDM) fiber-optic communications, network control and resourcemanagement issues in optical communication networks, and radio multipleaccess techniques. 


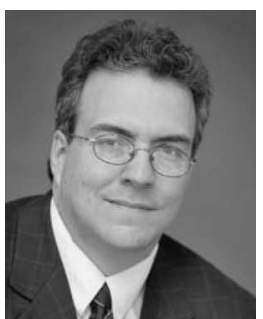

Paul Fortier (S'79-M'82-SM'00) received the B.Sc. and M.Sc. degrees in electrical engineering from Laval University, Quebec City, QC, Canada, in 1982 and 1984, respectively, and the M.S. degree in statistics and the Ph.D. degree in electrical engineering from Stanford University, Stanford, CA, in 1987 and 1989 , respectively.

Since 1989, he has been with the Department of Electrical and Computer Engineering, Laval University, where he is currently a Full Professor. From 1991 to 1997, he was the Program Director for the Department of Computer Engineering, and from 1997 to 2003, he was the Chairman of the Department of Electrical and Computer Engineering. Since 2003, he has been the Associate Dean for Development and Research at the Faculty of Science and Engineering. He has done consulting work for several companies and government agencies in Canada. His research interests include digital signal processing for communications and the study of complexity and performance tradeoffs in hardware implementations, with applications in wireless and optical communications. He has been involved in the organization of national and international conferences and workshops in these fields.

Dr. Fortier is a Fellow of the Engineering Institute of Canada.

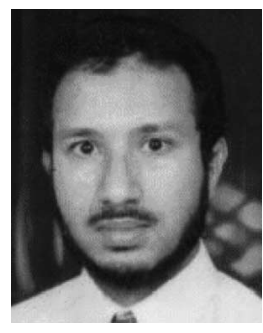

Hossam M. H. Shalaby (S'83-M'83-SM'99) was born in Giza, Egypt, in 1961. He received the B.S. and M.S. degrees from the University of Alexandria, Alexandria, Egypt, in 1983 and 1986, respectively, and the Ph.D. degree from the University of Maryland, College Park, in 1991, all in electrical engineering.

In 1991, he joined the Department of Electrical Engineering, University of Alexandria, as an Assistant Professor. He was promoted to Associate Professor in 1996 and to Professor in 2001 (current position). From March to April 1996, he was a Visiting Professor at the Electrical Engineering Department, Beirut Arab University, Beirut, Lebanon. From September 1996 to January 1998, he was an Associate Professor at the Electrical and Computer Engineering Department, International Islamic University Malaysia, and from February 1998 to December 1998, he was with the School of Electrical and Electronic Engineering, Nanyang Technological University, Singapore, where he was a Senior Lecturer, and from January 1999 to February 2001, an Associate Professor. Since December 2000, he has been an Adjunct Professor with the Department of Electrical and Information Engineering, Faculty of Sciences and Engineering, Laval University, Quebec City, QC, Canada. His research interests include optical communications, optical code division multiple access (CDMA), spread-spectrum communications, and information theory.

Dr. Shalaby received an Systems Research Center (SRC, MD) fellowship from 1987 to 1991, a State Award (Academy of Scientific Research and Technology, Egypt) in 1995 and 2001, and a University Award in 1996 (University of Alexandria, Egypt). He has served as a Student Branch Counselor (Alexandria University), IEEE Alexandria and North Delta Subsection, since 2002, and served as the Chairman of the Student Activities Committee of IEEE Alexandria Subsection from 1995 to 1996. He has also served as a Technical Referee for the Proceedings of the Institution of Electrical Engineers, the IEEE TRANSACTIONS ON COMMUNICATIONS, IEEE TRANSACTIONS ON INFORMATION THEORY, IEEE JOURNAL ON SELECTED AREAS IN COMMUNiCATIONS, and the IEEE JOURNAL OF LIGHTWAVE TECHNOLOGY. He is listed in the 14th edition of Marquis Who's Who in the World, 1997. 